globulin mucin 3 (TIM-3) or other checkpoints, ${ }^{4}$ and recently identified mechanisms of primary resistance to PD-1-PD-L1 blockade, which include transcriptional signatures in the tumor microenvironment (innate anti-PD-1 resistance signature, or IPRES ${ }^{5}$ ) and the presence of somatic differences in tumor cells (constitutive activation of $\beta$-catenin ${ }^{6}$ or loss of $\mathrm{PTEN}^{7}$ ) that inhibit the activation and recruitment of $\mathrm{T}$ cells to the tumor microenvironment.

Together, these observations shed further light on the possible mechanisms of treatment failure in patients who do not have a response to the current checkpoint antibodies, beyond the limited ability of the current Food and Drug Administration-approved PD-L1 companion immunohistochemical assays to identify patients who will or will not have a response. ${ }^{8}$ At the same time, the results also raise some important questions. Are they applicable to PD-1 resistance in other tumor types? Are there rational approaches to salvage therapy, such as the activation of downstream interferon pathways by cytosolic double-stranded DNA, which directly activates the stimulator of interferon genes (STING) ${ }^{9}$ in a JAK-independent fashion and could remedy some of the immune escape mechanisms detailed in the article? And could the mutated B2M-induced lack of expression of MHC class I be overcome by MHC class II-mediated CD4 T-cell recognition of tumor epitopes or by cross-presentation by stromal myeloid cells, leading to bystander elimination of antigen-loss variants? $?^{10}$

The ability of whole-exome sequencing to identify mechanisms of both innate and acquired resistance has potential clinical applications. As our knowledge of the host-tumor interactions at both the genomic and biologic levels increases, we inch closer to the time when extensive genomic analysis coupled with immune profiling will be applied to patients with cancer at the time of diagnosis and at relapse, to aid in selection of the combination therapy that is most likely to bring about eradication of an individual patient's tumor - the ultimate goal of precision medicine.

Disclosure forms provided by the authors are available with the full text of this editorial at NEJM.org.

From the Providence Cancer Center, Portland, OR.

This editorial was updated on September 1, 2016, at NEJM.org.

1. Zaretsky JM, Garcia-Diaz A, Shin DS, et al. Mutations associated with acquired resistance to PD-1 blockade in melanoma. N Engl J Med 2016;375:819-29.

2. Tumeh PC, Harview CL, Yearley JH, et al. PD-1 blockade induces responses by inhibiting adaptive immune resistance. Nature 2014;515:568-71.

3. D'Urso CM, Wang ZG, Cao Y, Tatake R, Zeff RA, Ferrone S. Lack of HLA class I antigen expression by cultured melanoma cells FO-1 due to a defect in B2m gene expression. J Clin Invest 1991;87:284-92.

4. Koyama S, Akbay EA, Li YY, et al. Adaptive resistance to therapeutic PD-1 blockade is associated with upregulation of alternative immune checkpoints. Nat Commun 2016;7:10501.

5. Hugo W, Zaretsky JM, Sun L, et al. Genomic and transcriptomic features of response to anti-PD-1 therapy in metastatic melanoma. Cell 2016;165:35-44.

6. Spranger S, Bao R, Gajewski TF. Melanoma-intrinsic $\beta$-catenin signalling prevents anti-tumour immunity. Nature 2015;523:231-5.

7. Peng $\mathrm{W}$, Chen JQ, Liu C, et al. Loss of PTEN promotes resistance to T cell-mediated immunotherapy. Cancer Discov 2016;6: 202-16.

8. Ma W, Gilligan BM, Yuan J, Li T. Current status and perspectives in translational biomarker research for PD-1/PD-L1 immune checkpoint blockade therapy. J Hematol Oncol 2016;9:47.

9. Corrales L, McWhirter SM, Dubensky TW Jr, Gajewski TF. The host STING pathway at the interface of cancer and immunity. J Clin Invest 2016;126:2404-11.

10. Spiotto MT, Rowley DA, Schreiber H. Bystander elimination of antigen loss variants in established tumors. Nat Med 2004;10: 294-8.

DOI: 10.1056/NEJMe1606042

Copyright (c) 2016 Massachusetts Medical Society.

\title{
Safety of Long-Acting Beta-Agonists in Children with Asthma
}

\author{
Andrew Bush, M.B., B.S., M.D., and Urs Frey, M.D., Ph.D.
}

Has there ever been more of a Jekyll-and-Hyde protein than the airway $\beta_{2}$ receptor? Short-acting beta-agonists give rapid relief from bronchospasm, but their overuse is a factor in asthmarelated deaths, and the less-selective agents have been associated with epidemics of patients dying during acute asthma attacks. In children with uncontrolled asthma, the Best Add-on Therapy Giving Effective Responses (BADGER) study showed that the addition of long-acting beta- 
agonists (LABAs) was more effective than increasing the dose of inhaled glucocorticoids in controlling asthma, ${ }^{1}$ but the accompanying editorial $^{2}$ highlighted the limited evidence regarding the safety of this approach and instead favored increasing the dose of inhaled glucocorticoids. It was clear that further safety studies were needed if the BADGER approach were to be adopted.

Thus, it is reassuring to see in the Journal a report of a large, well-conducted trial involving children that was mandated by the Food and Drug Administration (FDA) and that shows no excess of serious asthma events in children receiving a combination inhaler containing fluticasone propionate and salmeterol. ${ }^{3}$ Interestingly, as with the corresponding trial involving adults, ${ }^{4}$ there was some evidence of efficacy in terms of secondary outcomes for LABAs. The obvious strengths of the trial are the large numbers studied (>3000 participants in each group) and the reassuringly low number of adverse events. Although intercenter differences in treatment policies and definitions of asthma attacks are always difficult to control for, it is hard to imagine a better trial being performed in the near future. There are weaknesses in the trial; although it is large and well done, it does not completely rule out an adverse effect from LABAs. However, it does go a long way toward ruling out a class effect of these medications, but the findings should not be extrapolated uncritically to other medications in this class.

No clinical trial is perfect, and the trial conducted by Stempel et al. is no exception to that rule. To achieve a "real-world setting," medication use at baseline was not optimized. It may be that some children were being overtreated, and therefore the safety of LABAs in these children was in the context of overtreatment with inhaled glucocorticoids, and the conclusion might be different with an appropriate dose. In addition, this trial was predicated on the assumption that the risk factors for asthma-related death (the most pressing safety concern) and asthma attacks are the same. Exacerbations are related to asthma-control status, history of exacerbations, environmental triggers, and seasonal, genetic, and immunologic modifying risks, ${ }^{5}$ but the predominant causes of asthma-related death $^{6}$ are lack of access to health care $(45 \%$ of the participants had not attempted to seek pro- fessional help), lack of personal action plans, underuse of glucocorticoids, overuse or inappropriate use of bronchodilators, underestimation of asthma severity by treating doctors and by parents, lack of objective measures of airway obstruction, and nonadherence to the regimen, including drug and alcohol use by caretakers. Particularly in children and young people, the poor recognition of the risk of an adverse outcome was an important avoidable factor. The present trial controlled for many of these factors, and given the rarity of asthma-related deaths, a vastly larger study, which is unlikely ever to be practicable, would be needed if death was the end point. Nonetheless, a trial in which (we are happy to note) there were no asthma-related deaths in either group cannot rule out a small effect on asthma-related deaths that might have appeared in a much larger study.

What are the implications of this report for clinical practice? Clearly, the safety of a medication is not necessarily the best indication for its prescription. Most children will have their asthma controlled by low-dose inhaled glucocorticoids if taken regularly through an appropriate device. If asthma is not controlled, rather than uncritically adding on further therapies, pediatricians are advised to first check the adequacy of technique with the medication-delivery device, make sure that patients and children understand treatment and action plans, and at a minimum, verify whether the family is collecting enough prescriptions to cover the need for regular medications - something achieved by only one in six families in one study.

There is no evidence for the use of a combined inhaler as first-line preventive therapy in children, and this fact needs to be emphasized because such use is increasingly creeping into practice. ${ }^{8,9}$ Monotherapy with a LABA in a child should be considered medical negligence, and we suggest that single LABA inhalers should carry a warning to that effect, as required in the United States by the FDA in 2010. ${ }^{10}$ However, for the unusual child with asthma who needs more than low-dose inhaled glucocorticoids to control the disease or who has persistent, objectively documented, variable airflow obstruction, the present trial provides reassuring evidence that combination inhalers containing a LABA and an inhaled glucocorticoid are safe. 
Disclosure forms provided by the authors are available with the full text of this editorial at NEJM.org.

From the Department of Pediatric Respiratory Medicine, Royal Brompton Hospital, London (A.B.); and the Department of Pediatrics, University Children's Hospital Basel, University of Basel, Basel, Switzerland (U.F.).

1. Lemanske RF Jr, Mauger DT, Sorkness CA, et al. Step-up therapy for children with uncontrolled asthma receiving inhaled corticosteroids. N Engl J Med 2010;362:975-85.

2. von Mutius E, Drazen JM. Choosing asthma step-up care. N Engl J Med 2010;362:1042-3.

3. Stempel DA, Szefler SJ, Pedersen S, et al. Safety of adding salmeterol to fluticasone propionate in children with asthma. N Engl J Med 2016;375:840-9.

4. Stempel DA, Raphiou IH, Kral KM, et al. Serious asthma events with fluticasone plus salmeterol versus fluticasone alone. N Engl J Med 2016;374:1822-30.

5. Cook J, Saglani S. Pathogenesis and prevention strategies of severe asthma exacerbations in children. Curr Opin Pulm Med 2016;22:25-31.

6. National Review of Asthma Deaths. Why asthma still kills. London: Royal College of Physicians, August 11, 2015 (https:// www.rcplondon.ac.uk/projects/outputs/why-asthma-still-kills).

7. Warner JO. Review of prescribed treatment for children with asthma in 1990. BMJ 1995;311:663-6.

8. Bisgaard H, Szefler S. Long-acting beta 2 agonists and paediatric asthma. Lancet 2006;367:286-8.

9. Sweeney J, Patterson CC, O'Neill S, et al. Inappropriate prescribing of combination inhalers in Northern Ireland: retrospective cross-sectional cohort study of prescribing practice in primary care. Prim Care Respir J 2014;23:74-8.

10. FDA announces new safety controls for long-acting beta agonists, medications used to treat asthma. Silver Spring, MD: Food and Drug Administration, 2010 (http://www.fda.gov/ NewsEvents/Newsroom/PressAnnouncements/ucm200931.htm).

DOI: 10.1056/NEJMe1608508

Copyright (c) 2016 Massachusetts Medical Society.

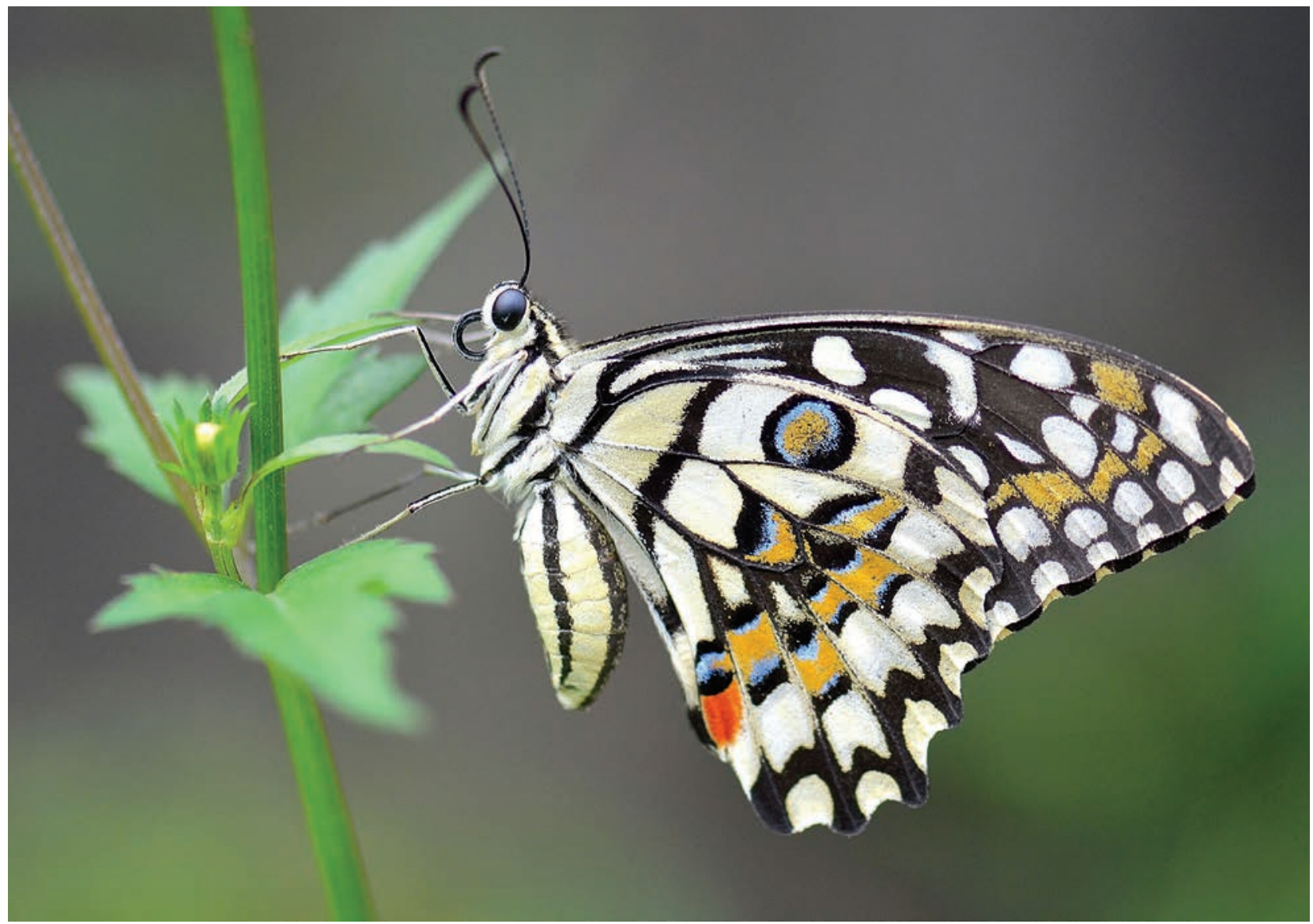

Papilio demoleus (Common Lime Butterfly)

Abhilash Pillai, M.D.

The New England Journal of Medicine

Downloaded from nejm.org at IMPERIAL COLLEGE LONDON on September 19, 2016. For personal use only. No other uses without permission. Copyright (C) 2016 Massachusetts Medical Society. All rights reserved. 